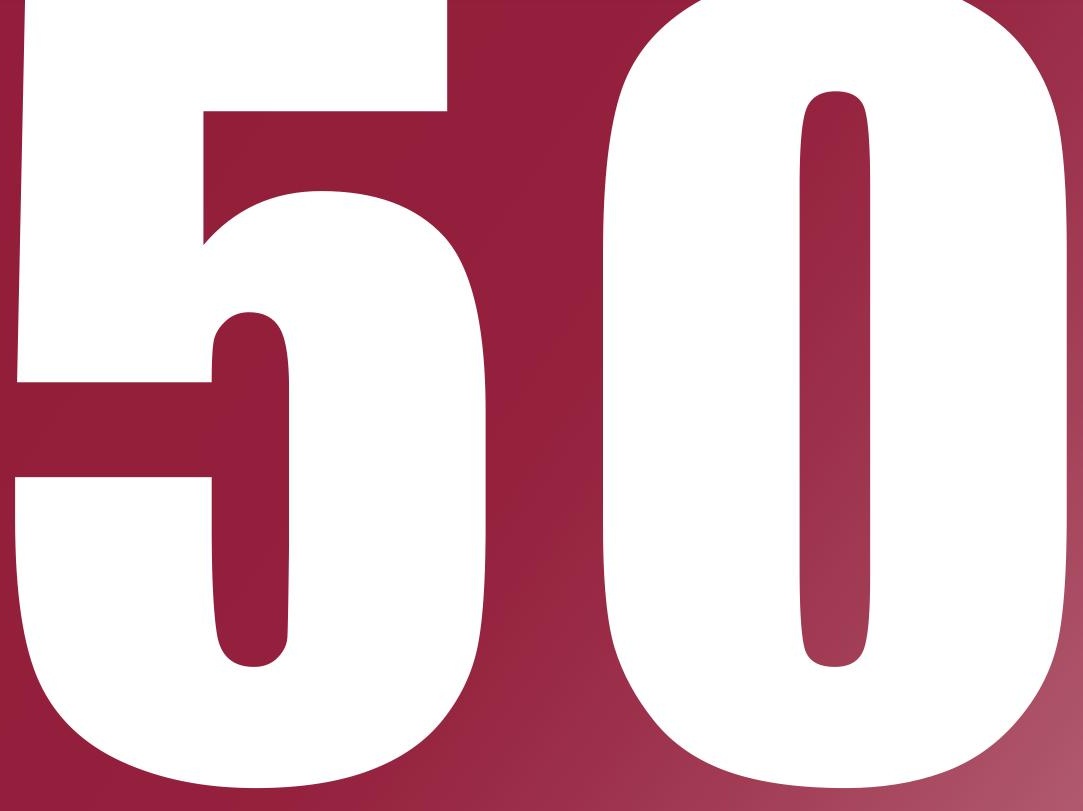

ÁMBITOS

REVISTA

INTERNACIONAL

DE

COMUNICACIÓN

$\mathbf{N}^{\circ} \mathbf{5 0}$

EDICIÓN OTOÑO

2020

ISSN: 1139-1979

E-ISSN: 1988-5733

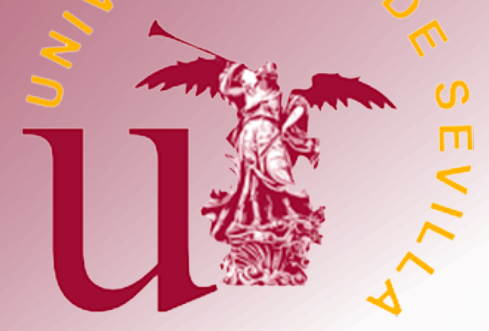




\section{INDICE}

EDITORIAL EDITORIAL

Presentación del Monográfico. Comunicación y juventud. Nuevos medios, representación, recepción y participación en contenidos de entretenimiento e información

Presentation of the monograph. Communication and youth. New media, representation, reception and participation in entertainment and information content.

Juan Francisco Gutiérrez Lozano, Francisco Javier Ruiz del Olmo

7-9

\section{MONOGRÁFICO MONOGRAPH}

El consumo audiovisual de la Generación Z. El predominio del vídeo online sobre la televisión tradicional The audiovisual consumption of Generation $Z$. The predominance of online video over traditional television

La imagen de los jóvenes en las estrategias transmedia de las series de televisión: el caso de Skam España en Instagram

The image of young people in transmedia strategies of TV series: the case of Skam España on Instagram

Sergio Jesús Villén Higueras, Francisco Javier Ruiz del Olmo

La penetración de Netflix en el público español ¿cuestiona el modelo televisivo tradicional?

Does the arrival of Netflix in the Spanish audience challenge the traditional television model?

Javier Bustos Díaz

49-61

Generación Z y consumo de información política: entre la televisión y los nuevos formatos mediáticos Generation $Z$ and consumption of political information: between television and new media formats

Ruth Gómez de Travesedo Rojas, Marta Gil Ramírez

$62-79$

Actualidad y comedia: El éxito de El Intermedio y La Resistencia entre los espectadores jóvenes

Information and comedy: The Success of El Intermedio and La Resistencia for young audiences

Inmaculada Concepción Aguilera García

80-95 
El efecto socializador de articular un espacio de comunidad virtual en el ecosistema del aula

The socializing effect of creating a virtual community space in the classroom environment

Lorea Ariadna Ruiz Gómez, Mónica Hinojosa Becerra, Francisco Javier Ruiz San Miguel

Juventude ciberativista e educação: reflexões sobre um jeito hacker de ser

Cyber activity youth and Education: reflections on a hacker way of being

Carla Azevedo de Aragão, Pietro Matheus Bompet Fontoura Alves, Karina Moreira Menezes

La risa grotesca en la obra de Bob Dylan: análisis de la Bacanal y el Festín Carnavalesco en la trilogía folk-rock eléctrica

Grotesque Laughter in Bob Dylan's Work: Analysis of The Bacchanal and the Carnival Feast in the Electric Folk-rock Trilogy

Jesús Albarrán Ligero

El papel del intertexto en el videojuego. Una partida, mil y una historias

Intertext in video games. A game, thousand and one stories

Rocío Serna-Rodrigo

145-158

\section{ÁmBitos PERSONALES PERSONAL ÁmBITOS}

El auge de Twitch: nuevas ofertas audiovisuales y cambios del consumo televisivo entre la audiencia juvenil The rise of Twitch: New audiovisual offers and the transformation of Television consumption among young audiences

\section{ARTÍCULOS ARTICLES}

La recepción de los medios de comunicación para residentes rusos en la Costa del Sol

Mass media reception by Russian residents on the Costa del Sol

Natalia Meléndez Malavé, José Carlos Pozo García

176-192

La calidad periodística en la cobertura de terremotos: Caso Ecuador

Journalistic quality in earthquake coverage: The case of Ecuador

Juan Pablo Arrobo-Agila, Mendoza María, Ignacio Aguaded

La credibilidad publicitaria en la nueva esfera mediática: los universitarios y los medios

Advertising credibility in the new media sphere: university students and the media 
Arco iris en medios brasileños: percepciones sociales sobre una campaña publicitaria LGBTQIA+

Rainbow in Brazilian media: social perceptions of an LGBTQIA+ advertising campaign

Leonardo Ferreira Batista, José Geraldo de Araújo Ramalho Filho

224-239

Rituales y comunicación política: la toma de posesión de los presidentes autonómicos españoles

Rituals and political communication: the Spanish autonomous Presidents inauguration

Ricardo Domínguez García

240-258

RESEÑAS REVIEWS

Una mirada diferente para hacer periodismo

A different look for making journalism

Guillermo Paredes-Otero

259-261

Periodismo y tecnología: una simbiosis continua

Journalism and technology: a continuous symbiosis

Luisa Graciela Aramburú Moncada

262-265

Una propuesta metodológica para impartir la asignatura de redacción periodística

Journalism and technology: a continuous symbiosis

Noelia Ruiz-Alba

266-268 


\title{
La credibilidad publicitaria en la nueva esfera mediática: los universitarios y los medios
}

\section{Advertising credibility in the new media sphere: university students and the media}

\author{
Noemí Martín García \\ Universidad de Valladolid| Plaza de la Universidad, no1.40005 Segovia| España \\ | http://orcid.org/0000-0003-3478-5021| noemicarmen.martin@uva.es
}

\section{Belén Ávila Rodríguez de Mier}

ESIC Business y Marketing School | Avenida de Valdenigrales s/n, Pozuelo de Alarcón. 28223

Madrid | España| Orcid: https://orcid.org/0000-0001-8381-4461| belen.avila@esic.edu

Fechas | Recepción: 05/05/2020 | Aceptación: 19/08/2020 | Publicación final: 15/10/2020

\section{Resumen}

La irrupción de internet y el avance de la tecnología han generado grandes cambios en la manera en que la población consume los medios de comunicación. Para la publicidad, este hecho ha suscitado un gran desafío al multiplicarse, muy rápidamente, los medios en los que los individuos pueden ser impactados por los mensajes comerciales. Este trabajo parte de la idea de que la confianza de los consumidores es clave para conseguir una publicidad eficaz (Adlantina, 2013). El objetivo es conocer la credibilidad que otorgan los universitarios de campus españoles a los mensajes comerciales emitidos en los diferentes medios. Para ello se realizaron 604 encuestas a jóvenes universitarios de 18 a 24 años. Los resultados muestran como los mensajes publicitarios emitidos en medios convencionales como la prensa, la radio o la televisión cuentan con una mayor credibilidad

\section{Abstract}

The irruption of the internet and the advancement of technology has generated great changes in the way the population consumes the media. For advertising, this fact has created a great challenge as the media in which individuals can be impacted by commercial messages multiply very quickly. This work starts from the idea that consumer confidence is key to achieving effective advertising (Adlantina, 2013). The objective is to find out the credibility that Spanish university students give to commercial messages broadcast in different media. To do this, 604 surveys were conducted for young university students between the ages of 18 and 24 . The results show how the messages broadcast in the 'conventional media' such as the press, radio or television have greater credibility than those broadcast on the 'new channels' such as email, influencers or social

Forma de citar:

Martín García, N.; Ávila Rodríguez de Mier, B. (2020). La credibilidad publicitaria en la nueva esfera mediática: los universitarios y los medios. Ámbitos. Revista Internacional de Comunicación 50, pp. 208-223. doi: 
que los emitidos en nuevos canales como el correo electrónico, los influencers o las redes sociales. La investigación también señala que el género no influye en la credibilidad otorgada a la publicidad, pero si lo hace el área de conocimiento y el gusto por la publicidad. Y que los medios con más audiencia no tienen por qué ser los más creíbles. Este estudio pretende servir de ayuda a los profesionales de la comunicación en la toma de decisiones estratégicas de medios más eficaces.

Palabras clave: credibilidad, jóvenes, publicidad, audiencia, medios de comunicación. networks. The research also indicates that gender does not influence the credibility granted to advertising but does the field of studies and the taste for the publicity of respondents. And that the media with the most audience does not have to be the most credible. This study aims to help communication professionals in strategic media decision for the most effective media.

\section{INTRODUCCIÓN}

El aumento del número de canales de comunicación producido por la irrupción de internet y el avance de las Tecnologías de la Información y Comunicación (TIC) están generando importantes cambios en el panorama mediático. De ahí que el análisis de la percepción de la credibilidad que se tiene sobre los medios y las noticias sea un tema de gran interés tanto para el campo académico como para el profesional. Prueba de ello son las investigaciones sobre la credibilidad del contenido informativo de los medios de Calvo-Porral, Catalina-García, GarcíaJiménez y Montes (2015), Espinosa, Ochaíta y Gutiérrez-Rodríguez (2014), García-Avilés, Navarro-Maíllo y Arias-Robles (2014), Martínez-Fernández y Juanatey-Boga (2014), Rastrollo (2010), Roses y Farias (2012) o Turcotte, York, Irving, Scholl y Pingre (2015).

Sin embargo, pese a que el sector publicitario gestionó en medios más de 12.835,5 millones de euros en el año 2018 (Infoadex, 2018), apenas existen estudios específicos sobre la percepción de la credibilidad del mensaje publicitario (Ávila Rodríguez-de-Miery Martín-García, 2019; Del Barrio, 2002 y Zapata y Martínez-Caro, 2016).

Este trabajo se realiza con objeto de conocer el grado de credibilidad que otorgan los jóvenes universitarios a los mensajes comerciales emitidos en los diferentes medios de comunicación, así como para descubrir posibles diferencias en función del género, el área de conocimiento o el gusto por la publicidad.

El motivo de centrar la investigación en los jóvenes universitarios es diverso. Por un lado, se trata de un "colectivo clave para poder comprender los cambios culturales, de costumbres y de actitudes que se van produciendo en el devenir de los pueblos, en un contexto progresivamente globalizado" (Ballesta, Gómez, Lozano, Guardiola y Serrano, 2003 p.247). La manera en que los jóvenes se relacionan con los medios muestra una tendencia que, probablemente, marque el futuro del sector de la comunicación (García-Jiménez, Tur-Viñes y Pastor, 2018). Este grupo ha crecido en un "contexto de tecnologías relacionales y de 
comunicaciones digitales" (Buckingham y Martínez-Rodríguez, 2013, p.12), por lo que su análisis es de gran relevancia para prever los futuros movimientos del sector. Por otro lado, los estudiantes universitarios poseen el perfil más consumidor de internet de la sociedad española (Observatorio Nacional de las Telecomunicaciones y de la Sociedad de la Información, 2018) y representan una nueva forma de consumo de medios ya que, además de ser nativos digitales (Prensky, 2001), el hecho de estar cursando estudios superiores les "obliga a convivir con medios, predominantemente interactivos, no sólo como elementos de ocio, sino también como herramientas de trabajo dado el actual proceso de convergencia en el que se halla envuelta la universidad española" (Iglesias y González-Díaz, 2012). Por ultimo, señalar que los jóvenes conforman un grupo sobre el que las marcas comerciales ejercen un gran poder de seducción, llegando a darse un vínculo entre el consumo de determinadas marcas y la sensación de "bienestar subjetivo" (Vargas Bianchi, 2019, p.166).

La importancia de este estudio radica en que, en un entorno tan saturado de medios y mensajes comerciales, la credibilidad se postula como la base para conseguir una publicidad eficaz (Adlantina, 2013). Por tanto, el conocer algo más de la percepción de la credibilidad publicitaria entre los universitarios ayudará a los profesionales de la comunicación en la toma de decisiones estratégicas de medios eficaces. Para Paz Aparicio, Vázquez Casielles y Santos Vijande (2000), la eficacia publicitaria es un "elemento clave para determinar si se han logrado o no los objetivos establecidos, calcular la rentabilidad de esas inversiones, y asegurar con una mayor probabilidad el éxito de campañas futures" (Paz Aparicio, Vázquez Casielles y Santos Vijande, 2000, p.5)

\section{LA PERCEPCIÓN DE LOS JÓVENES Y LA CREDIBILIDAD}

La manera en que la población consume los medios de comunicación ha experimentado grandes cambios estructurales y de contenido. Este hecho responde tanto a la irrupción de internet como al avance de las tecnologías que facilitan el acceso a la información en cualquier momento y desde múltiples lugares y dispositivos. Este nuevo entorno, mucho más competitivo, ha supuesto que medios consolidados como la prensa y las revistas impresas pierdan adeptos a favor de su versión online (AIMC, 2019; La publicidad, 2019; Larrañaga, 2008) más abierta a un ecosistema mundial (Peña, Lazkano y García-González, 2016) o que el visionado de television desde los canales convencionales se este reduciendo en favor de las plataformas digitales tales como Netflix o HBO (Del Pino y Aguado, 2012; Madinaveita y Merchante, 2015; Quintas-Froufe y González-Neira, 2016).

La publicidad no es ajena al nuevo contexto y prueba de ello es la investigación sobre la relación de los jóvenes con la publicidad online de Martinez-Costa, Serrano-Puche, Portada y Sánchez-Blanco (2019) en la que se manifiesta que si bien los jóvenes perciben como molesta la publicidad en el medio online, no utilizan los bloqueadores para prescindir de ella. Muela y Baladrón (2010) investigan las percepciones que los jóvenes tienen de la publicidad online y concluyen que el reto de los anunciantes sigue siendo "entablar a través de sus acciones de comunicación relaciones basadas en la confianza y en la transparencia" (Muela y Baladrón, 2010, p.195). 
Por otro lado, empiezan a ser recurrentes las investigaciones centradas en las redes sociales. Entre ellas destaca la de De Frutos, Petrel y Sánchez-Valle (2014) que concluye que los jóvenes muestran "buena predisposición a relacionarse con las marcas en su propio espacio de la red social, en contraste con la publicidad de Internet" (De Frutos, Petrel y Sánchez Valle, 2014, p.69) o la de Llorente, Bartolomé y Navarro (2013) en donde se analiza la marca Mango y su uso de Facebook como plataforma de publicidad de sus productos. Aparte de las anteriores, se localizan investigaciones sobre lo que está suponiendo el nuevo entorno digital en los medios convencionales. Un ejemplo es el trabajo de Guerrero y Crespo (2020) sobre el desgaste de la publicidad televisiva entre los jóvenes ecuatorianos o el estudio de Neuromedia (2018) en el que se analiza el recuerdo publicitario en los medios en diferentes tramos de edad para concluir que "la edad afecta al recuerdo. A mayor edad menor recuerdo publicitarios" y que "la televisión y exterior son los medios más recordados en todas las edades" (Neuromedia, 2018).

Partiendo del nuevo escenario de consumo de medios, las agencias de publicidad a través de la planificación estratégica, deciden cuáles son los medios y soportes más óptimos para publicar las comunicaciones de las distintas marcas (Ávila Rodríguez-de-Mier Martín-García, 2017; De Frutos, 2019; González-Lobo, Carrero-López y Mariñas-González, 2018). Estos canales se seleccionan, principalmente, en función de los datos de audiencia suministrados por las diferentes fuentes de medición -Kantar Media, ComScore o el Estudios General de Medios (EGM)-. A la hora de realizar esta elección es donde entra en juego la credibilidad tanto de la fuente como del mensaje en la medida en que:

Si un determinado mensaje publicitario es percibido como muy poco creíble por parte de la audiencia, ello va a afectar negativamente a la aceptación del mensaje y, por ende, a la marca. Por tanto, los anunciantes deben huir de aquellas fórmulas publicitarias que sean consideradas por los consumidores como poco creíbles. (Del Barrio, 2002, p.129).

Por ello, conocer la percepción de la credibilidad que tienen los consumidores de los mensajes publicitarios emitidos en los diferentes medios ayudará a la hora de desarrollar estrategias de comunicación más eficaces.

La credibilidad es el resultado de un procedimiento cognitivo en el que un individuo examina y evalúa, de manera subjetiva, las características que tiene un emisor, un contenido, una fuente o cualquier otro elemento informativo (Austin y Dong, 1994; Choi, Watt y Lynch, 2006; Gunther, 1992; Hovland y Weiss, 1951; Metzger, Flanagin y Zwarum, 2003; Meyen y Schwer, 2007; Roses y Gómez-Calderón, 2015; Slater y Rouner, 1996). De ahí que los consumidores tengan una mayor probabilidad de elegir marcas que consideran creíbles ya que el riesgo de compra es percibido como menor (Erdem y Swait, 2004). Las primeras clasificaciones para medir la credibilidad de la información suministrada por los medios surgieron en el ámbito anglosajón en la segunda mitad del siglo XX (Gaziano y MaGranth, 1986; Meyer, 1988; Newell y Goldsmith, 2001; Oyedi, 2008). Estudios que se han multiplicado desde la llegada de internet y las nuevas tecnologías (Calvo-Porral, Martínez-Fernández y Juanatey-Boga, 2014; Espinosa, Ochaíta y Gutiérrez-Rodríguez, 2014; Rastrollo, 2010; Roses y Farias, 2012; o Turcotte, York, Irving, Scholl y Pingre, 2015). Algunos de ellos centrados en el público joven como son el de 
Catalina-García, García-Jiménez y Montes (2015) en el que se analizan una serie de medios de comunicación convencionales y su versión online para afirmar que las informaciones más creíbles son las emitidas en la radio online y las menos creíbles las emitidas por las redes sociales o el de García-Avilés, Navarro-Maíllo y Arias-Robles (2014) donde se concluye que los jóvenes dan más credibilidad a las noticias recibidas a través de los medios sociales.

Estos cuantiosos estudios se reducen significativamente cuando la investigación se centra en la información publicitaria. En el ámbito académico encontramos el estudio de Ávila Rodríguezde-Mier y Martín-García (2019) sobre la influencia que ejerce tanto el emisor de la publicidad como el canal de emisión en la percepción de credibilidad publicitaria del público senior. En este trabajo se concluye que los mensajes publicitarios en radio y los anuncios institucionales son los que suscitan una mayor credibilidad entre los más mayores. Otro de los estudios es el realizado por Del Barrio (2002) donde se manifiesta que los consumidores consideran menos creíbles los mensajes basados en la publicidad comparativa. Por último, cabe destacar el trabajo de Zapata y Martínez-Caro (2016) sobre la veracidad que otorga a los mensajes comerciales el uso de prescriptores. Dentro del campo profesional destaca, por un lado, el informe Global Trust in advertising de la consultora Nielsen (2015), en el que se da una visión de la confianza en la publicidad en diferentes canales y en diferentes públicos generacionales. En él se afirma que "la proliferación de los formatos de anuncios en internet no perjudica la confianza en los canales tradicionales de publicidad pagada" (Nielsen, 2015, p.2). Y, por otro lado, está el estudio realizado por la agencia Giovanni+Draftdcb (2014) enfocado en la neurociencia y con el objetivo de buscar una mejor comunicación entre marcas y consumidores con base en la confianza. No obstante, ninguno de estos trabajos se focaliza en el segmento joven y/o universitario.

\section{OBJETIVOS Y METODOLOGÍA}

Esta investigación parte de la idea de que la persuasión depende, entre otros factores, de la credibilidad de la fuente (Aristóteles, 1954 citado por Oyedi, 2010) y de que, debido a los cambios en el panorama mediático, este criterio se postula como la base para conseguir una publicidad más eficaz (Adlantina, 2013). El objetivo principal es conocer el grado de credibilidad que le otorgan los universitarios a los mensajes comerciales emitidos en los diferentes medios de comunicación publicitaria. Dicho objetivo se concreta en tres objetivos secundarios que son:

- $\quad$ Conocer si existen diferencias por género en relación a la credibilidad que otorgan los universitarios a los mensajes comerciales emitidos en los diferentes medios de comunicación publicitaria.

- Descubrir la vinculación existente entre la credibilidad que otorgan los jóvenes universitarios a los mensajes comerciales emitidos en los diferentes medios de comunicación publicitaria y su área de conocimiento (estudios relacionados con la comunicación comercial o estudios ajenos a esta). 
- Averiguar cómo incide la variable 'gusto por la publicidad' en la credibilidad que otorgan los universitarios a los mensajes comerciales emitidos en los diferentes medios de comunicación publicitaria.

Para ello, se realizó un estudio de tipo descriptivo y correlacional con el método de la encuesta basada en las directrices de Wimmer y Dominick (1996). Se trabajó con una muestra de conveniencia por cercanía formada por un total de 604 individuos con edades comprendidas entre los 18 y 24 años - edad máxima considerada por la Unesco para delimitar a los jóvenes (Unesco, s.f.)-. La edad media fue de 20.24 años, la desviación típica de 1.726 y la mediana de 20 años. La premisa que imperó a la hora de formar la muestra fue que el 50\% de los encuestados tenían que cursar estudios relacionados con el campo de la comunicación comercial $(n=302)$ mientras que el otro $50 \%(n=302)$ no tenían que tener ninguna relación con esta disciplina. Para la variable género la muestra se dividió en un $70.7 \%$ mujeres $(n=407)$ y $29.3 \%$ hombres ( $n=177$ ). Finalmente, para la variable 'gusto por la publicidad', la muestra se filtró mediante una pregunta del cuestionario sobre el gusto por la publicidad. Los resultados de esta variable mostraron que a un $48 \%$ Si les gustaba la publicidad $(n=283)$, a un $42 \% \mathrm{~A}$ veces $(n=248)$ y un $10 \%$ declaró no gustarle $(n=58)$.

Los cuestionarios fueron suministrados en el campus de Segovia de la Universidad de Valladolid -grados de Publicidad y Relaciones Públicas (RR.PP.), Magisterio e Ingeniería Informática-, en la Universidad de Extremadura - grado de Veterinaria- y, finalmente, en ESIC Business y Marketing School Madrid- grados de Publicidad y RR.PP. y de Administración y Dirección de Empresas- y se cumplimentaron de manera voluntaria. Como se apuntaba al inicio del artículo, la elección de estudiar a los estudiantes universitarios se debió a tres motivos. El primero, los jóvenes representan un grupo clave para conocer los nuevos comportamientos sociales y su relación con los medios es tendencia (Ballesta et al., Buckingham y Martínez-Rodríguez, 2013 y García-Jiménez, Tur-Viñes y Pastor, 2018). El segundo, los estudiantes universitarios posen el perfil más consumidor de internet de la sociedad española (ONTSI, 2018) además de representar una nueva forma de consumir medios (Iglesias y González-Díaz, 2012). Por ultimo, los jóvenes universitarios conforman un grupo de alto valor comercial para las marcas y se encuentran en las puertas de formar parte del tan deseado target comercial.

Para dar respuesta a los objetivos se realizaron cinco preguntas. La primera de ellas era abierta - P1: Edad-y las tres siguientes fueron cerradas de selección multiple, P2: Género - con dos respuestas posibles: Hombre o Mujer-, P3: ¿Estudias alguna carrera relacionada con la comunicación? -con dos respuestas posibles: Si o No- P4: ¿Te gusta la publicidad? - con cuatro respuestas posibles: Si, No, A veces y No sabe/No contesta- P5: ¿Cómo de creíble consideras la publicidad recibida a través de los siguientes medios? Televisión, radio, prensa en papel, revistas en papel, exterior, cine, medios de comunicación digitales, correo electrónico, influencers, redes sociales y blogs. Esta última pregunta tenía cuatro respuestas posibles: Muy creíble, Creíble, Nada creíble o No sabe/No contesta. Los medios analizados corresponden a los utilizados por Infoadex en su estudio sobre la inversión publicitaria en España (Infoadex, 
2018). A excepción del medio online que, debido a los cambios que se han producido en la manera de hacer publicidad y la importancia adquirida en las fases de investigación, estratégica y planificación (Papí et al., 2016), se ha segmentado en cinco canales diferentes medios de comunicación digitales, correo electrónico, influencers, redes sociales y blogs - y del medio suplementos dominicales dada la dificultad para diferenciarlo del resto de revistas impresas.

El trabajo de campo se desarrolló en los meses de marzo y abril del 2019. El modo de reparto fue doble, por un lado, a través de un formulario auto-administrado vía Google Forms y, por otro lado, impreso en papel. El tiempo de realización no superó, en ninguno de los casos, los cinco minutos.

El análisis de los resultados se llevó a cabo sobre los porcentajes de respuesta obtenidos y en base al cálculo del valor de la credibilidad. Este último se ha obtenido a través de la realización de una escala de intervalo específica cuyos valores oscilan de 1 a 3, siendo 1 nada creíble, 2 creíble y 3 muy creíble. Las respuestas "no sabe/ no contesta" no se han contemplado para no desvirtuar los resultados. Para analizar las variables de género, área de conocimiento y gusto por la publicidad se ha utilizado el análisis comparativo de medias mediante la distribución t de Student con un nivel de significación para $p<.05$.

\section{RESULTADOS}

Los datos en torno a la credibilidad que le otorgan los universitarios a los mensajes comerciales emitidos en los diferentes medios (Gráfico 1 ) sitúan a casi todos por debajo del valor medio de la credibilidad (2.0). A pesar de ello, la prensa papel (2.0) junto con la radio (1.9) son los medios que cuentan con una mejor valoración de sus mensajes comerciales. Por el contrario, los medios digitales como el correo electrónico (1.4), los influencers (1.5) y las redes sociales (1.5) son los canales con una publicidad peor valorada. Los resultados muestran como 7 de cada 10 jóvenes considera 'nada creíble' la publicidad recibida a través del correo electrónico (67.9\%). Y a más de la mitad de ellos les sucede lo mismo con los influencers y las redes sociales ( $57.6 \%$ y $53.9 \%$ ). Por el contrario, 8 de cada 10 universitarios considera 'creíble' o 'muy creíble' la publicidad emitida en radio (78.9\%) y en la prensa en papel (78.8\%). 


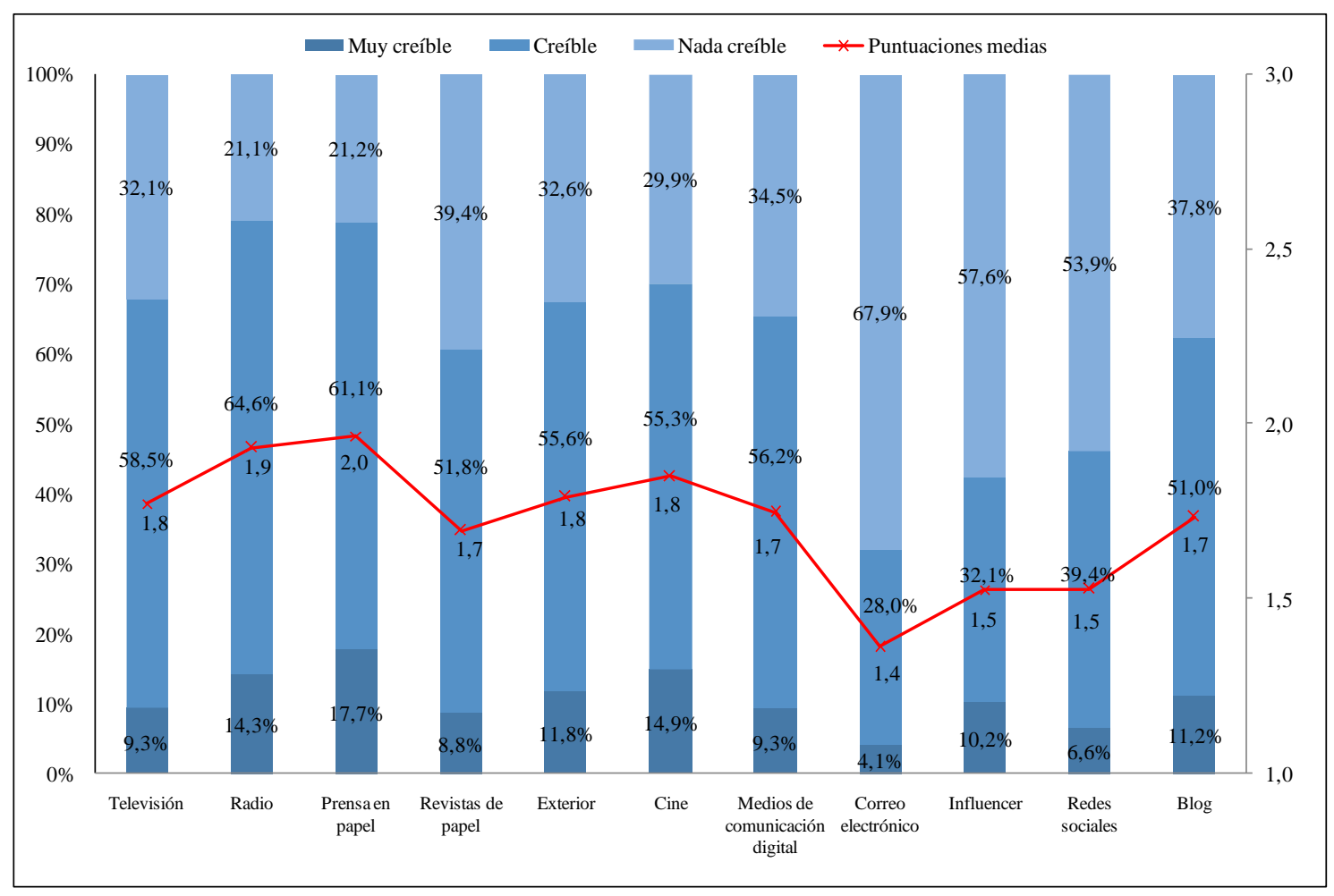

Gráfico 1. Credibilidad de la publicidad en los diferentes medios

Fuente: Elaboración propia.

\subsection{Incidencia de la variable 'genero'}

Los resultados en relación a la influencia de la variable género muestran como este no es un indicador diferencial en cuanto a la credibilidad que otorgan los universitarios a la publicidad emitida en los diferentes medios (Tabla 1). Las puntuaciones medias de credibilidad son iguales para ambos (1.8). Las pequeñas diferencias reseñables indican que las féminas se comportan de manera algo más crédula ante la publicidad del medio exterior (1.7 H vs. $1.8 \mathrm{M}$ ) y los mensajes recibidos a través del correo electrónico $(1.3 \mathrm{H}$ vs. $1.4 \mathrm{M})$. Por el contrarío, los hombres se muestran más confiados con los anuncios de cine (1.9 H vs. $1.8 \mathrm{M})$, influencers (1.6 $\mathrm{H}$ vs. $1.5 \mathrm{M})$ y blogs $(1.8 \mathrm{H}$ vs. $1.7 \mathrm{M})$. La distribución t de Student confirmó que no existen diferencias significativas entre la credibilidad y el género en ningún medio a excepción del correo electrónico donde la comparación de las puntuaciones medias si es significativa $(p=0.015)$ mostrándose las féminas más crédulas con los mensajes comerciales emitidos por este canal.

Tabla 1.

Puntuaciones medias valor credibilidad por género

\begin{tabular}{|l|c|c|c|}
\hline & Credibilidad Hombres & Credibilidad Mujeres & Dif. H. M \\
\hline Televisión & 1.8 & 1.8 & 0.0 \\
\hline Radio & 1.9 & 1.9 & -0.1 \\
\hline
\end{tabular}




\begin{tabular}{|l|c|c|c|}
\hline Prensa en papel & 2.0 & 2.0 & 0.0 \\
\hline Revistas en papel & 1.7 & 1.7 & 0.1 \\
\hline Exterior & 1.7 & 1.8 & -0.1 \\
\hline Cine & 1.9 & 1.8 & 0.0 \\
\hline Medios comunicación digitales & 1.7 & 1.8 & -0.1 \\
\hline Correo electrónico & 1.3 & 1.4 & -0.1 \\
\hline Influencers & 1.6 & 1.5 & 0.1 \\
\hline Redes sociales & 1.5 & 1.5 & 0.0 \\
\hline Blogs & 1.8 & 1.7 & 0.1 \\
\hline
\end{tabular}

Fuente: Elaboración propia.

\subsection{Incidencia de la variable 'área de conocimiento'}

Los datos relativos a la credibilidad otorgada a la publicidad según el área de conocimiento de los encuestados (estudios relacionados o no con la comunicación comercial) muestran, en general, como los estudiantes de Publicidad y RR.PP. atribuyen una mayor credibilidad a la publicidad emitida en los medios objeto de estudio (1.9) que los que cursan grados ajenos a esta disciplina (1.6) (Gráfico 3). A pesar de ello, ninguno considera que estos mensajes sean creíbles por encima del valor medio otorgado (2.0). Por medios, los estudiantes de comunicación comercial consideran más creíble la publicidad en todos los medios a excepción del correo electrónico donde ambos se muestran igual de desconfiados (1.4 en ambos grupos). Los datos también señalan como las diferencias entre la percepción de la credibilidad de los que estudian Publicidad y RR.PP. y del resto son menores en la radio y la prensa en papel (0.1 punto de diferencia) mientras que aumentan cuando se les pregunta por los medios digitales 0 el exterior (0.4 puntos de diferencia). La comparación de puntuaciones medias corrobora la existencia de diferencias significativas en todos los medios excepto el correo electrónico donde la $t$ de Student confirma que no existen diferencias en cuanto a la credibilidad otorgada por los universitarios a la publicidad recibida en el correo electrónico en función del área de conocimiento que cursen $(p=0.722)$. 


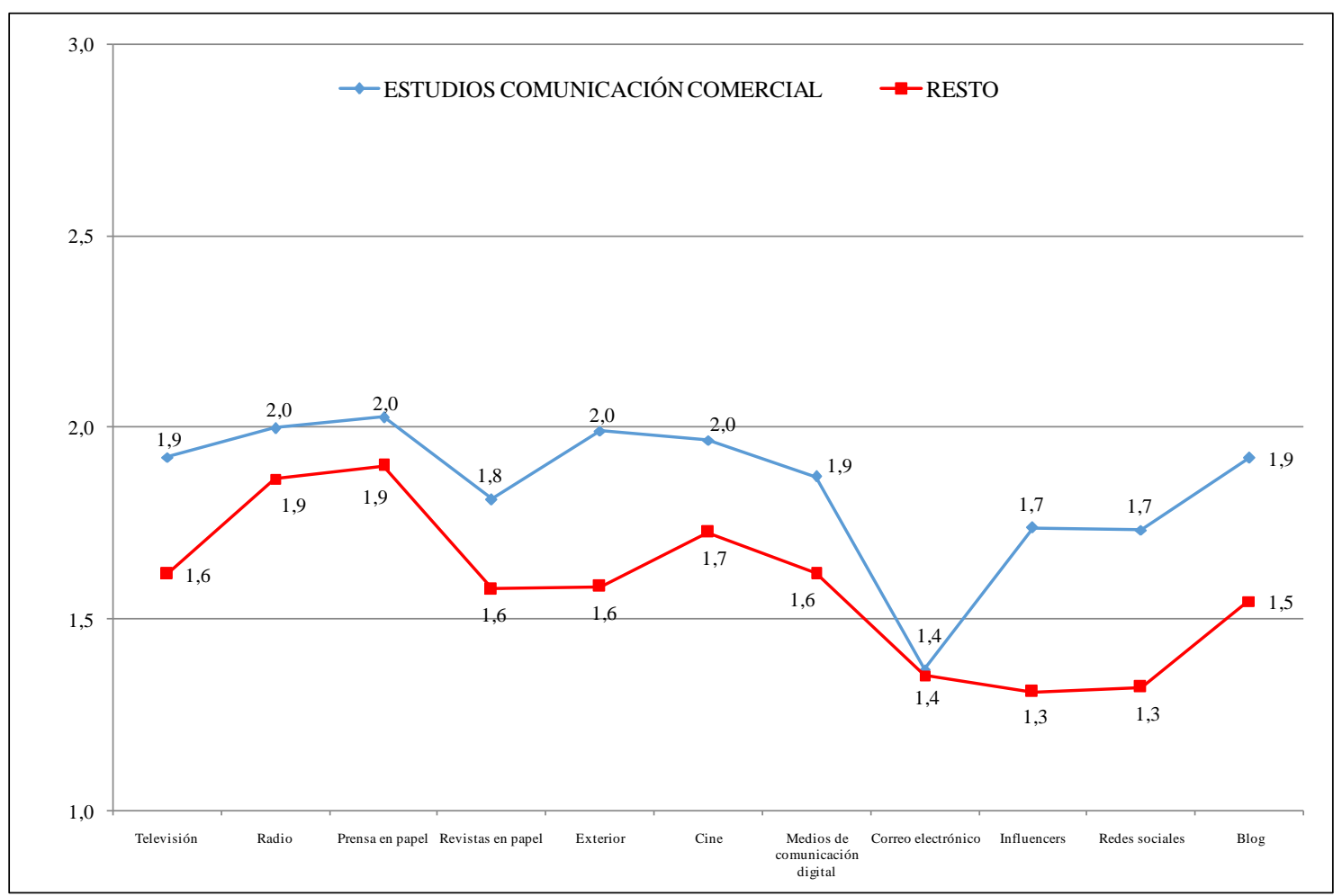

Gráfico 2. Valor de la credibilidad por medio y por área de conocimiento Fuente: Elaboración propia.

\subsection{Incidencia de la variable 'gusto por la publicidad'}

Por último, los datos sobre la incidencia de la variable 'gusto por la publicidad' muestran que existe una relación entre el interés manifestado por la publicidad y la credibilidad que se otorga a los anuncios emitidos en los medios. En general, la credibilidad en la publicidad es mayor en aquellos encuestados que manifestaron que les gustaba la publicidad -Si 1.8, A veces 1.7 y No gusta 1.4-. Por medios, en el Gráfico 4 se puede ver que el correo electrónico seguido de la radio son los medios donde esa diferencia entre el gusto por la publicidad y la credibilidad es menor - diferencia entre variables gusta, no gusta y a veces en el correo electrónico 0.2 y en radio de $0.6-$. En la radio y en los blogs es donde menos incidencia tiene la variable gusto por la publicidad - diferencia entre variables gusta, no gusta y a veces en el exterior 1.2 y los blog 1.1-. La distribución t de Student demostró que existen diferencias significativas en relación a la credibilidad con la variable gusto o no gusto por la publicidad en todos los medios, con excepción del correo electrónico en el que la credibilidad no está condicionada por el gusto por la publicidad $(p=0.163)$ 


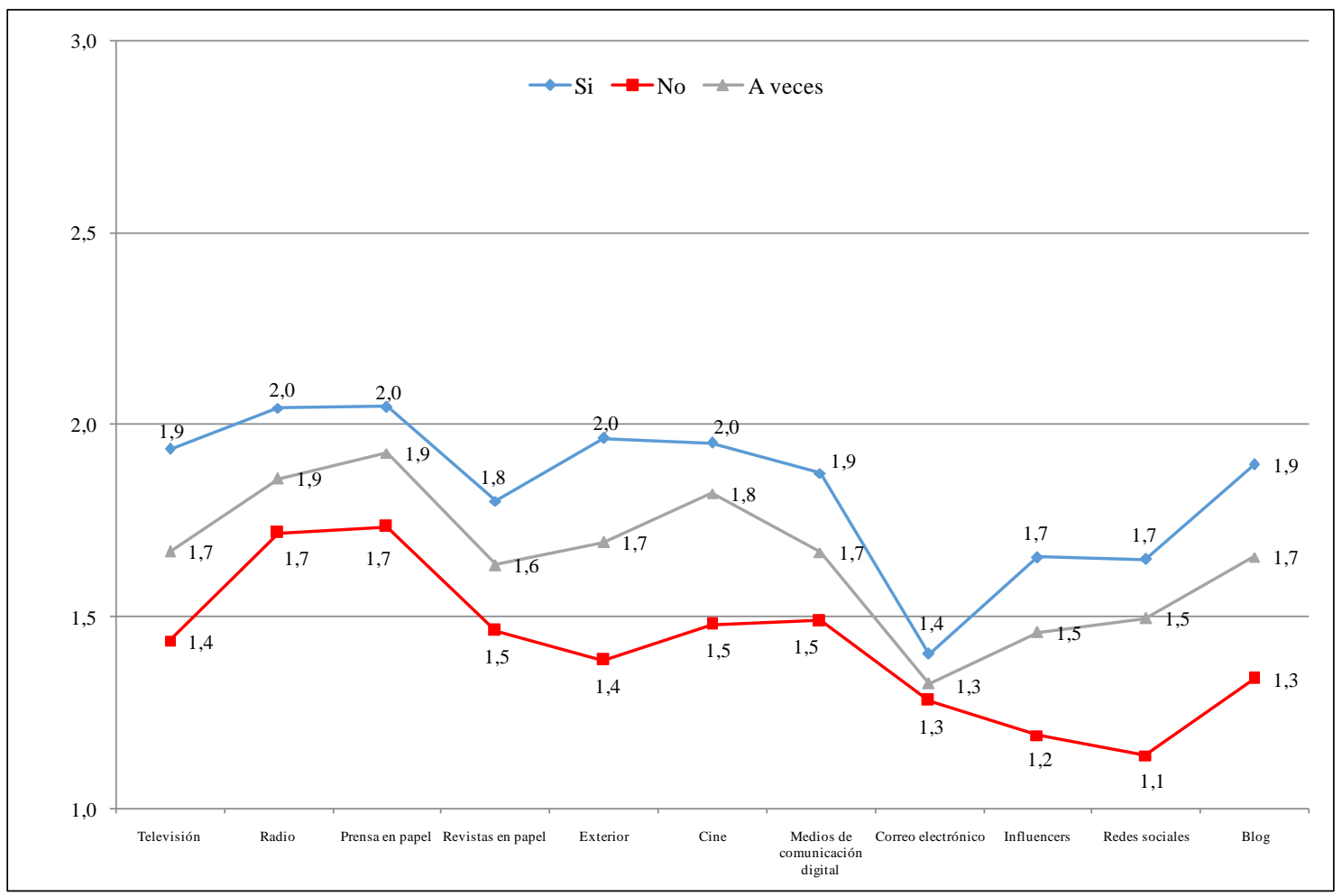

Gráfico 3. Valor de la credibilidad por medio en función de su gusto por la publicidad Fuente: Elaboración propia

\section{CONCLUSIÓN Y DISCUSIÓN}

El estudio constata la tendencia de que los universitarios confian más en la publicidad emitida en los medios convencionales como la prensa o la radio que en la emitida a través de medios digitales como el correo electrónico o los influencers. Estos datos vienen a corroborar los resultados del estudio Navegantes en la Red (AIMC, 2019) en el que se afirma que al $63.4 \%$ de los usuarios del medio online les molesta más la publicidad en internet que en el resto de los medios y que el $62.8 \%$ considera que se siente perseguido por los anuncios digitales. Por tanto y en base a ambos estudios, se podría afirmar que, según sostiene Dirconfidencial (2018), "la percepción de la publicidad en internet por parte de los usuarios es muy negativa". Estos resultados están en línea con los trabajos de Flanagin y Metzer (2010), Hilligoss y Rieh (2007), Metzger, Flanagin y Zwarun (2003) y Parratt (2010), citados por García, Tur-Viñes y Pastor (2018) para los que la televisión, la prensa impresa y la radio tienen una mayor credibilidad que el medio online.

Los resultados del presente estudio se acentuan al compararse con los índices de audiencia de los estudiantes universitarios de 18 a 24 años suministrados por el EGM (Tabla 2). Cuando unimos ambos datos, se observa que los medios de mayor audiencia son los que cuentan con un menor índice de credibilidad publicitaria y viceversa, los que tienen los anuncios más creíbles son los menos consumidos por estos jóvenes. Este es el caso de la publicidad en la prensa impresa que con el mayor grado de credibilidad (2.0) presenta uno de los porcentajes 
de penetración sobre los univesitarios más bajos (15.01\%). En este sentido también destacan las redes sociales y el correo electronico donde, a pesar la poca credibilidad con la que cuenta su publicidad (1.5), destacan por su gran audiencia $(77.8 \%$ redes sociales y $72.2 \%$ correo electrónico).

Tabla 2. Credibilidad vs. audiencia por medios

\begin{tabular}{|l|c|c|}
\hline & Puntuación media valor credibilidad & \% Audiencia \\
\hline Prensa en papel & 2.0 & 15.01 \\
\hline Radio & 1.9 & 59.21 \\
\hline Cine & 1.8 & 9.91 \\
\hline Exterior & 1.8 & 86.95 \\
\hline Televisión & 1.8 & 78.76 \\
\hline Medios comunicación digitales & 1.7 & 52.24 \\
\hline Blogs & 1.7 & 3.48 \\
\hline Revistas en papel & 1.7 & 36.5 \\
\hline Redes sociales & 1.5 & 77.79 \\
\hline Correo electrónico & 1.5 & 72.24 \\
\hline
\end{tabular}

Fuente: Elaboración propia (valor de la credibilidad) y EGM, 2019 (audiencia).

En este sentido también apuntan las conclusiones del estudio de Nielsen (2015) en el que se indica que, para la Generación Z (15-20 años) y la generación millennial (21-34 años), los anuncios en televisión, revistas y periódicos gozan de una mayor confiabilidad que los expuestos en el correo electrónico o las redes sociales (Nielsen, 2015). A su vez, estas conclusiones se vinculan con las aportaciones del estudio de Neuromedia (2018) donde se sostiene que el $90 \%$ de los jóvenes recordó los anuncios publicitarios de la televisión y que la radio y la prensa poseen el recuerdo más cualificado gracias a "su baja saturación, la frecuencia de la exposición a la publicidad y la credibilidad que el propio usuario les otorga" (Neuromedia, 2018).

Otra de las conclusiones del presente estudio es que no existen diferencias por género respecto a la credibilidad que se otorga a los mensajes comerciales emitidos en los diferentes medios, pero si en relación al área de conocimiento y a su gusto por la publicidad. Los estudiantes de comunicación comercial se muestran más confiados con la publicidad que aparece en los medios que los que no estudian esta disciplina. Teniendo en cuenta que según los datos elaborados por el Ministerio de Ciencia, Innovación y Universidades solo el $5.5 \%$ de los estudiantes están matriculados en alguna carrera que tiene que ver con la comunicación 
comercial (Ministerio de Ciencia, Innovación y Universidades, 2019), este estudio implica que se tienen que buscar nuevas formas de publicidad que aumenten la credibilidad de los mensajes comerciales y, por ende, los haga más eficaces. En este mismo sentido se manifiesta Castelló (2010, p.94) al afirmar que "en la actualidad, y frente al descenso que sufre la publicidad convencional en términos de credibilidad, está demostrado que la alternativa más fiable para una buena estrategia de marketing es la generación de recomendaciones entre consumidores, a través de estrategias de Marketing Participativo". La tercera de las variables analizadas en el estudio verificó que existe una relación entre el gusto por la publicidad y la credibilidad que le otorgan a los anuncios emitidos en los medios. Por tanto, las campañas que gusten a los consumidores serán consideradas más creíbles y, por ende, como afirmaba Adlantina (2013), más eficaces. Es importante destacar que el correo electrónico es el único medio analizado que se comporta diferente al resto de canales y en el que la credibilidad de los anuncios si varía en función del género del entrevistado y no en función del campo de estudio y gusto por la publicidad.

Una de las limitaciones del estudio es que la muestra no es representativa de los universitarios que estudian en campus españoles, pero sí ofrece resultados interesantes y novedosos que abren la puerta a futuras investigaciones más amplias. A pesar de ello, el estudio plantea una nueva visión de los medios en el entorno de la planificación y la creación de estrategias publicitarias, poniendo de relieve que no solo las audiencias son importantes para la selección de emplazamientos, sino que se tiene que tener en cuenta otras variables como la credibilidad para poder establecer engagement con el consumidor.

\section{Referencias}

Adlantina (2013). La credibilidad es la clave para lograr una publicidad efectiva. Recuperado de https://bit.ly/35tl7ko

AIMC (2019). Infografía Resumen 21 ํN Navegantes en la red. Recuperado dehttps://bit.ly/2Wsnr8m

Austin, Erica y Dong, Qingwen. (1994). Source v. content effects on judgments of news elievability. Journalism quarterly, 71(4), 973-983.doi:10.1177/107769909407100420

Ávila Rodríguez-de-Mier, B. y Martín-García, N. (2019). La población senior y su percepción de la publicidad: influencia del medio y del emisor. SEECI, (49), 19-37. doi:10.15198/seeci.2019.49.19-37

Ávila Rodríguez-de-Mier, B. y Martín-García, N. (2017). Plan de seguimiento y control en medios. En J. Alard y A. Monfort (coord.) Plan de comunicación on y off en la práctica, (pp. 211-234). Madrid: ESIC Editorial.

Ballesta, F. J., Gómez, J.A., Lozano, J., Guardiola, P. y Serrano, F. (2003). El consumo de medios en los jóvenes de Secundaria. Madrid: Editorialccs

Buckingham, D. y Martínez-Rodríguez, J.B. (2013). Jóvenes interactivos: Nueva ciudadanía entre redes sociales y escenarios escolares. Comunicar, 20(40), 10-14.doi:10.3916/C40-2013-02-00

Choi, J., Watt, J.y Lynch, M. (2006). Perceptions of news credibility about the war in Iraq: Why war opponents perceived the internet as the most credible medium. Journal of computer-mediated communication, 12, 209-229. doi:10.1111/j.1083-6101.2006.00322.x 
Catalina-García, B., García-Jiménez, A. y Montes, M. (2015). Jóvenes y consumo de noticias a través de Internet. Historia y Comunicación Social, 20(2) ,601-619. doi:10.5209/rev_HICS.2015.v20.n2.51402

Castelló, A. (2010). Estrategias empresariales en la Web 2.0. Alicante: ECU.

Calvo-Porral, C., Martínez-Fernández, V. A. y Juanatey-Boga, O. (2014). Credibilidad de los medios de comunicación: análisis de la prensa diaria desde el comportamiento del consumidor. El profesional de la información, 23 (3), 300-309. doi:10.3145/epi.2014.may.10

Calvo-Porral, C., Martínez-Fernández, V.A. y Juanatey-Boga, O. (2014). La credibilidad de los medios de Comunicación de masas: una aproximación desde el Modelo de Marca Creíble Intercom. Revista Brasileira de Ciências da Comunicação, 37(2), 21-49. doi:10.1590/1809-584420141.

De Frutos, B. (2018). Los medios publicitarios: Investigación, planificación y gestión. Madrid: Editorial Síntesis.

De Frutos, B., Petrel, M. y Sánchez Valle, M. (2014). La interacción de los jóvenes con las marcas en las redes sociales: hacia la presencia consentida y deseada. AdComunica, 7, 69-86. doi: http://dx.doi.org/10.6035/2174-0992.2014.7.5

Del Barrio, S. (2002): La credibilidad como elemento clave en la eficacia de la publicidad comparativa en prensa: un estudio experimental, Investigaciones Europeas de Dirección y Economía de la Empresa, 8(1) ,119-134. Recuperado de https://bit.ly/3fjt5IX

Del Pino, C. y Aguado, E. (2012). Internet, Televisión y Convergencia: nuevas pantallas y plataformas de contenido audiovisual en la era digital. El caso del mercado audiovisual online en España. Observatorio (OBS*) Journal, 6(4), 057-075. doi:10.15847/obsOBS642012590

Dirconfidencial (2018). La saturación publicitaria se mantiene como el mayor problema de Internet. Recuperado de https://bit.ly/3flUH9S

Erdem, T.y Swait, J. (2004). Brand Credibility and its role in brand choice and consideration. Journal of Consumer Research, 31, 191-199. doi:10.1086/383434

Espinosa, M.A., Ochaíta E. y Gutiérrez-Rodríguez H. (2014). Adolescentes consumidores de televisión: autopercepciones sobre sus derechos. Comunicar,42(23), 181-188. doi:10.3916/C43-2014-18

García-Avilés, J. A., Navarro-Maíllo, Fy Arias-Robles, F. (2014). La credibilidad de los contenidos informativos en internet para los 'nativos digitales': estudio de caso. Palabra Clave, 17(3), 875894. doi:10.5294/ pacla.2014.17.3.13

García-Jiménez, A., Tur-Viñes, V. y Pastor, Y. (2018). Consumo mediático de adolescentes y jóvenes. Noticias, contenidos audiovisuales y medición de audiencias. Icono 14, 16(1), 22-46. doi:10.7195/ri14.v16i1.1101

Gaziano, C. y Mcgranth, K. (1986). Measuring the Concept of credibility. Journalism Quarterly, 63 (3), 451-462.doi:10.1177/107769908606300301

Giovanni+ Draftdcb (2014). Trustparency study. Recuperado de https://bit.ly/2Yx2xrt

González-Lobo, M.A., Carrero-López, E. y Mariñas-González, G. (2018). Manual de planificación de medios: todo lo que hay que saber para planificar correctamente los medios. Madrid: ESIC Editorial.

Guerrero, K.A. y Crespo, V. (2020). La obsolescencia de la publicidad televisiva entre los jóvenes ecuatorianos, caso de estudio RTS. Redmarka: revista académica de marketing aplicado, 24, (1), 106-116. doi.org/10.17979/redma.2020.24.1.6459

Gunther, A. (1992). Biased press or biased public? Attitudes toward media coverage of social groups. The public opinion quarterly, 56(2), 147-167. Recuperado de https://bit.ly/3drtZez 
Hovland, C.I. y Weiss, W. (1951). The influence of source credibility on communication effectiveness. Public Opinion Quarterly, 15, 635-650. doi:.1086/266350

Iglesias, M. y González-Díaz, C. (2012). Radiografía del consumo de medios de comunicación en estudiantes universitarios. Icono 14, 10(3), 100-115. doi:10.7195/ri14.v10i3.212

Infoadex (2018). Estudio de la inversión publicitaria en España 2018. Recuperado de https://bit.ly/2W1fOXo

La publicidad (2019). Cambios en el consumo de medios. Recuperado de https://bit.ly/2Ywlen5

Larrañaga, J. (2008). Análisis económico de la evolución de las audiencias de los medios tradicionales e impacto de internet en su consumo. Área Abierta, 21, 1-18. Recuperado de https://bit.ly/2zatINU

Llorente, C., Bartolomé, A. y Navarro, E. (2013). Eficacia publicitaria en redes sociales: el caso de Mango en Facebook España. Questiones publicitarias 18 (1), 93-110. Recuperado de https://ddd.uab.cat/pub/quepub/quepub_a2013n18/quepub_a2013n18p93.pdf

Madinaveita, E. y Merchante, M. (2015). Medición de audiencias: desafío y complejidad en el entorno digital. Harvard Deusto marketng y ventas, 131, 26-33. Recuperado de https://bit.ly/3dd8XQz

Martínez-Costa, M.P., Serrano-Puche, J., Portilla-Manjón, I. y Sánchez-Blanco, C. (2019). La interacción de los jóvenes adultos con las noticias y la publicidad online. Comunicar, (59), 19-28. doi:10.3916/C59-2019-02

Metzger, M. J., Flanagin, A. y Zwarum, L. (2003). College student Webc use, perceptions of information credibility, and verification behavior. Computers y Education 41(3), 271-290 doi:10.1016/S03601315(03)00049-6

Meyer, P. (1998). Defining and measuring credibility of newspapers: Developing an index. Journalism Quarterly, 65(3),567-574. doi:10.1177/107769908806500301

Meyer, P. (2004). The vanishing newspaper: Saving journalism in the information age. Columbia: University of Missouri Press.

Meyen, M. y Schwer, K. (2007). Credibility of media offering in centrally controlled media systems: a qualitative study bases on the example of East Germany. Media, culture y society, (29), 284303.doi:10.1177/0163443707074260

Ministerio de Ciencia, Innovación y Universidades (2019). Número de estudiantes egresados en Grado (1ㅇy 2 을 Ciclo). Recuperado dehttps://bit.ly/3fgolld

Muela, C. y Baladrón, A.J. (2010). Jóvenes y publicidad on line: nuevos espacios y formas, otros retos. Revista de Estudios de Juventud, 88, 183-199. Recuperado de http://www.injuve.es/sites/default/files/RJ88-13.pdf

Neuromedia (2018). Recuerdo publicitario por edad y medio. Recuperado de https://bit.ly/2L19lpo

Newell, S.J. y Goldsmith, R.E. (2001). The development of a scale to measure perceived corporate credibility. Journal of Business Research, 52(3), 235-247. doi: 10.1016/S0148-2963(99)00104-6

Nielsen (2015). Global Trust in advertising. Recuperado de https://bit.ly/35x5gmb

ONTSI (2018). Perfil sociodemográfico de los internautas, análisis de datos INE 2018. Observatorio nacional de las telecomunicaciones y de la Sociedad de la Información. Recuperado de https://bit.ly/2LYS6Wp

Oyedeji, T. (2008). The credible brand model: the effects of ideological congruency and customer-based brand equity on media and message credibility. University of Missouri. Recuperado de https://bit.ly/2zYeOuM 
Oyedeji, T. (2010). The credible brand model: the effects of ideological congruency and customer-based brand equity on new credibility. American Behavioral Scientist, 54(2), 83-99. doi:10.1177/0002764210376312

Papí, N., Iglesias, M., Penalva, C., González-Río, M.J., Orbea, J. y Ros, J. (2016). Publicidad en medios online: investigación, estrategia y planificación en J.D. Álvarez Teruel, S. Grau Company y M.T. Tortosa Ibáñez (coords.). Innovaciones metodológicas en docencia universitaria: resultados de investigación (pp. 549-557). Alicante: Instituto de ciencias para la innovaciónRecuperado de https://bit.ly/2zPNTkD

Paz Aparicio, C., Vázquez Casielles, R. y Santos Vijande, L. (2000). Publicidad y eficacia publicitaria influencia de la posición, repetición y estilos publicitarios en la eficacia de los anuncios televisivos entre los jóvenes. Documentos de trabajo (Universidad de Oviedo. Facultad de Ciencias Económicas), $203 . \quad$ Recuperado de https://dialnet.unirioja.es/servlet/articulo?codigo $=1252828$

Peña, S., Lazkano, I. y García-González, D. (2016). La transición digital de los diarios europeos: nuevos productos y nuevas audiencias. Comunicar, 24(46), 27-36.doi:10.3916/C46-2016-03

Prensky, M. (2001). Digital game-based learning. California: McGraw-Hill

Quintas-Froufe, N. y González-Neira, A. (2016). Consumo televisivo y su medición en España: camino hacia las audiencias híbridas. El profesional de la información, 25(3), 376-383. doi:10.3145/epi.2016.may.07

Ritacco, E. (2016). La saturación publicitaria, el gran enemigo de la publicidad. Adlatina. Recuperado de https://bit.ly/2xD7DaE

Rastrollo, M. Á. (2010). Credibilidad de la empresa informativa. Una perspectiva organizativa. En Actas II Congreso Internacional Latina de Comunicación Social, Universidad La Laguna. Recuperado de https://bit.ly/35sFyzg

Roses, S. y Farias, P. (2012). Credibilidad de los medios: un análisis bivariado de las opiniones de los españoles. Revista Mediterránea de Comunicación, 3(1), 79-104. doi: 10.14198/MEDCOM2012.3.06

Roses, S. y Gómez-Calderón, B. (2015). Credibilidad de los medios en España: divergencias de percepción y caracterización de los escépticos. El profesional de la información, 24 (4), 432-439. doi:10.3145/epi.2015.jul.10

Slater, M. D. y Rouner, D. (1996). How message evaluation and source attributes may influence credibility assessment and belief change. Journalism quarterly, 73(4), 974-991. doi:10.1177/107769909607300415

Turcotte J., York C., Irving J., Scholl R. M. y Pingre R. J., (2015). News Recommendations from Social Media Opinion Leaders: Effects on Media Trust and Information Seeking. Journal of ComputerMediated Communication, 20 (5), 520-535. doi:10.1111/jcc4.12127

Unesco (s.f.). La Unesco trabajando por y para los jóvenes. Recuperado de https://bit.ly/3fj3QjO

Vargas Bianchi, L. (2019). Incidencia del consumo y marcas en la experiencia de bienestar subjetivo entre jóvenes: una exploración cualitativa. Revista de Comunicación, 18(1), 166-190. doi:org/10.26441/RC18.1-2019-A9

Wimmer, R. D. y Dominick, J. R. (1996). La investigación científica de los medios de comunicación. Barcelona: Bosch.

Zapata, S. y Martínez-Caro, L. (2016). La influencia de la credibilidad de las celebridades en la publicidad. Anuario de Jóvenes Investigadores, 9, 240-243. Recuperado de https://bit.ly/2SwYkjt 